\title{
Hierarchical QoS-Aware Routing in Multi-tier Wireless Multimedia Sensor Networks
}

\author{
Stephane Lohier $^{1}$, Gilles Roussel ${ }^{1}$, and Yacine Ghamri Doudane ${ }^{2}$ \\ ${ }^{1}$ Université Paris-Est - IGM, 75420 Marne-la-Vallée Cedex - France \\ ${ }^{2}$ ENSIEE-LRSM, 18, allée Jean Rostand, 91025 Evry Cedex - France \\ lohier@univ-mlv.fr, roussel@univ-mlv.fr, ghamri@ensiie.fr
}

\begin{abstract}
The Wireless Multimedia Sensor Networks (WMSN) are a particular case of Wireless Sensors Networks (WSN) as they present a lower density, a limited mobility, require more important resources and need QoS control to transport the multimedia streams. In this paper, we propose, starting from a reference architecture of WMSN, a first approach for hierarchical selforganizing routing ensuring a certain level of QoS.
\end{abstract}

\section{Introduction}

The emergence and the development of WMSN [1] are related to the availability of low-cost and low-consumption CMOS cameras. For this new kind of networks the applications are numerous: monitoring of public or private places (concerts, borders, companies, houses...); detection, recognition and tracking of objects; automobile traffic management (speed control, car parking assistance...); industrial process control (visual inspection, automated actions...).

In these particular sensor networks, the main concerns are not only the scalability and the energy but also the QoS needed by multimedia streams: delay guarantees for the real time flows; bandwidth on the links related to the tolerable compression ratio; limited loss ratios. Besides, the routing problem on WSN [2] is different from that met on MANET (many-to-one and not many-to-many); the type of routing (hierarchical or data-centric) and the QoS metrics are strongly related to the application (application aware). Moreover, for WMSN, we have to consider specific factors: low mobility, low density, heterogeneous sensors, specific nodes capabilities for the processing and the storage.

Figure 1 described our proposal for a multi-tier architecture inspired by the work of I.F. Akyldiz [1]. In this heterogeneous and hierarchical architecture, each tier corresponds to a category of video sensors with increasing capabilities in term of camera resolution, processing, storage and transmission. For the first tier, the sensors can be CMUCam (weak resolution of 160x255) coupled with microcontrollers allowing a minimum processing and not very greedy transmissions like in ZigBee, Bluetooth or UWB standards. The second tier can be made up of webcam and microcontrollers with more processing, more storage and mixed transmissions, ZigBee and 802.11 for instance. The last tier is connected to the sink (multimedia server) and includes high resolution cameras coupled with laptop. An implementation example of this type of architecture is presented in [3]. 
For each tier, our proposal is to organize the topology in clusters with Cluster Head $(\mathrm{CH})$, Cluster Routers (CR) allowing multi-hop routing when necessary, and Cluster Terminals (CT), only able to capture video information and to transmit it. In order to limit the interferences, the nearby clusters can use distinct transmission channels. The sensors of the various levels can be moved but are not permanently mobile.

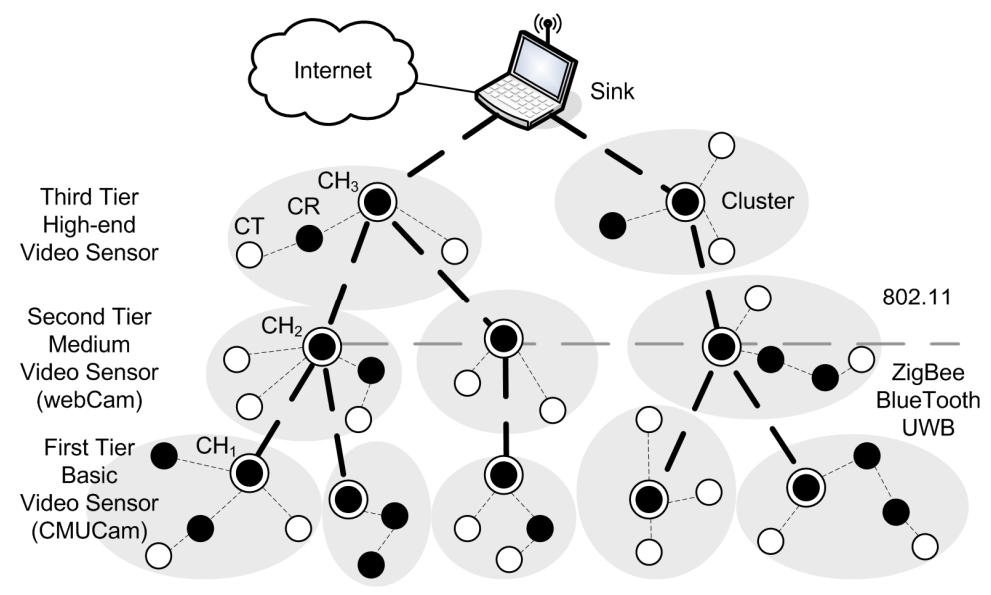

Fig. 1. Multi-tier Architecture of WMSN

Moreover, the processing, essentially carried out in the $\mathrm{CHs}$ and towards the collector, includes specifics operations like data aggregation (images from different scenes in the same flow) and data suppression (redundant images from various sensors). For all these reasons (clustered architecture, many-to-one flows, heterogeneous capabilities, processing into the $\mathrm{CHs}$ ) we believe that a hierarchical routing is the most suited.

The following sections present a first approach for QoS-aware hierarchical routing inside clusters, whatever is the tier, and between the clusters of the various tiers. The QoS routes setup is a first step of our solution. Indeed, the network organization must remain evolutionary according to the periodic requests of nodes to join/leave a cluster and to the needs of the sink-application which will select, starting from descriptors, interesting flows (image of a particular zone with a selected resolution...). Thus, the objective here is not to guarantee a QoS constantly but to choose and receive pictures of a sufficient quality (soft QoS) by dynamically optimizing the links quality, the processing in the nodes and the choice of the sources.

\section{Intra-cluster QoS Routing}

The proposed QoS routing in each cluster is proactive and includes 6 stages for the cluster self-organization and the routes setup procedures (figure 2):

1. Each node self-determines its possible role $(\mathrm{CH}, \mathrm{CR}$ or $\mathrm{CT})$ in a cluster according to fixed or periodically re-evaluated criteria: 
- sufficient storage and energy (comparison with specific thresholds for each role);

- for $\mathrm{CH}$ :

- transmission capacities (need for the corresponding interfaces);

- computation capacity for aggregation, suppression, compression....

2. Each $\mathrm{CH}$ initiates a cluster (scan channels, select a channel, select a cluster id...).

3. CR and CT carry out a research for a cluster:

- CR/CT broadcast a message for discovery: Cluster_Discovery_Request (Scan Channels...);

- response of the nearby CHs (and/or CR in case of multi-hop) with a Cluster_Discovery_Response (Cluster Description...).

4. Estimation of a cost for the concerned links (use of a function integrating the selected QoS metrics, see section 4) starting from the exchange of the Cluster_Discovery messages of stage 3 .

5. $\mathrm{CR}$ and $\mathrm{CT}$ choose a $\mathrm{CH}$ (or a $\mathrm{CR}$ ) according to the previous QoS estimate and join a cluster:

- CR/CT send a message Cluster_Join_Request (Clusterid...);

- response of the selected CH (or CR) with a Cluster_Join_Response (Cluster id, Network addresses...).

The associations of the CRs and the CTs are carried out in a recursive way: for the multi-hop routing, a $\mathrm{CT}$ out of the $\mathrm{CH}$ range has to wait for the association of a nearby CR to obtain an answer and thus to join in its turn a cluster.

6. The CR informs its $\mathrm{CH}$ (or its nearby $\mathrm{CR}$ which is closer to $\mathrm{CH}$ ) of its router's role:

- CR send a message Cluster_Router_Request (Cluster id...);

- Response of CH (or CR) with a Cluster_Router_Response (Cluster id, Network address block...). In its response, the $\mathrm{CH}$ specifies the address block (or the subblock for the response of a CR) which can be used by the CR for its CT (or its lower level CR).

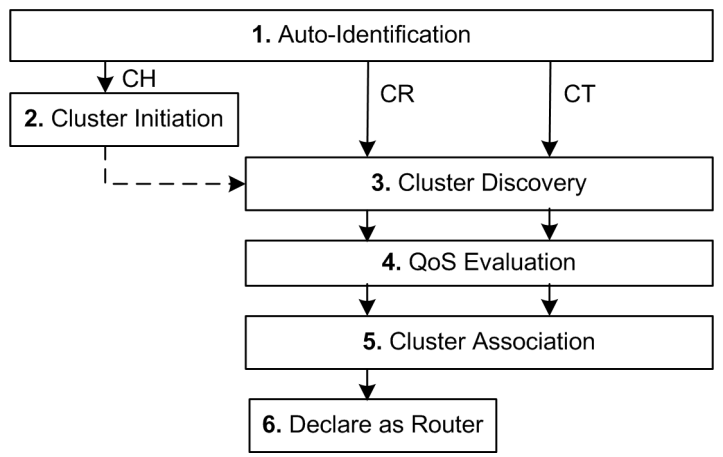

Fig. 2. Intra-cluster Routing Algorithm

After one or several exchange cycles, each CR or CT knows the address of its $\mathrm{CH}$ or its nearby $\mathrm{CR}$ (the one with the lower cost toward the $\mathrm{CH}$ ); each $\mathrm{CH}$ or $\mathrm{CR}$ has a routing table for all its nodes. The routing is hierarchical: all the data go towards $\mathrm{CH}$. According to the network dynamicity, the various stages are periodically re-launched. 


\section{Inter-tier QoS Routing}

The routing between the $\mathrm{CHs}$ of the various tiers (figure 1) proceeds in 5 stages according to the same hierarchical and recursive principle:

1. The $\mathrm{CH}_{1}$ ( $\mathrm{CH}$ of first tier) broadcast to know the $\mathrm{CH}_{2}$.

2. The $\mathrm{CH}_{1}$ choose a $\mathrm{CH}_{2}$ according to a cost estimated on the links (see section 4).

3. The $\mathrm{CH}_{2}$ broadcast in their turn on the corresponding interface to know the $\mathrm{CH}_{3}$.

4. The $\mathrm{CH}_{2}$ choose a $\mathrm{CH}_{3}$ according to a cost estimated on the links.

5. The $\mathrm{CH}_{3}$ broadcast to know the sink. In this last case, the cost is also computed to evaluate if, according to its position and its environment, the $\mathrm{CH}_{3}$ can obtain a sufficient QoS on the link towards the sink.

After sufficient exchanges, each $\mathrm{CH}_{\mathrm{i}}$ knows the address of its $\mathrm{CH}_{\mathrm{i}+1}$ or of the sink (transmitted with the broadcast response). Each node of each cluster can thus transmit towards the sink and conversely (the sink knows the $\mathrm{CH}_{3}$ which knows the $\mathrm{CH}_{2} \ldots$ ).

\section{Estimation of the Links Cost}

During the messages exchange of the discovery stage (between $\mathrm{CT} / \mathrm{CR}$ and $\mathrm{CR} / \mathrm{CH}$ or between $\mathrm{CH}_{\mathrm{i}}$ and $\mathrm{CH}_{\mathrm{i}+1}$ ), the cost on each possible links is periodically estimated and compared (i) with a threshold to decide if the node is under the conditions (range, noise, contentions...) to obtain a sufficient QoS and (ii) with the costs on the other links to choose the most efficient $\mathrm{CR} / \mathrm{CH}$. The cost function on a link between two nodes $\mathrm{i}$ and $\mathrm{j}$ ( $\mathrm{j}$ being the closest to the sink) can be expressed according to the chosen QoS metrics:

$$
C_{i j}=c_{1} \times f\left(\text { delay }_{i j}\right)+c_{2} \times f\left(\operatorname{SNR}_{j i}\right)+c_{3} \times f\left(e_{i j}\right)+c_{4} \times f\left(\text { energy }_{i}\right)+c_{5} \times f\left(\text { energy }_{j}\right)
$$

- $f\left(\right.$ delay $\left._{i j}\right)$ is a function of the delay for a data packet transmitted from i towards $\mathrm{j}$;

- $f\left(S N R_{j i}\right)$ is a function of the Signal/Noise Ratio measured from $\mathrm{j}$ towards $\mathrm{i}$;

- $f\left(e_{i j}\right)$ gives the error rate for the data packets transmitted from i towards $\mathrm{j}$;

- $f\left(\right.$ energy $\left._{i}\right)$ and $f\left(\right.$ energy $\left._{j}\right)$ give respectively the remaining energy in $\mathrm{i}$ and $\mathrm{j}$.

The choice of the coefficients $c_{1}$ to $c_{5}$ depends on the application and the type of traffic (the delay is more important than the loss rate for streaming...).

Besides, for "real time" applications, it is necessary to control the end-to-end delay. Rather than using higher level protocols like RTP and RTCP which involve overload, we can evaluate this global delay at the routing level, starting from the delay on each link and the knowledge of the route towards the sink, both information being provided by our routing protocol. The loss rate on a path from a CT towards the sink can also be evaluated, starting from the successive loss rates and the knowledge of the route.

\section{Conclusion}

In this paper, we presented a first approach for QoS routing in the WMSN. Insofar as the nodes are very few, not very mobile, and heterogeneous in term of resources, a 
hierarchical routing passing by "Cluster Head" is the most suitable. To introduce QoS, we proposed a cost function, estimated on a hop and which can be extended on several hops or the complete route towards the sink. The continuation of this study will be directed to (i) the complete specifications and the tests of the routing algorithm and the cost function, in connection with the possible MAC layers (ZigBee, UWB, 80211) and (ii) the specification of the data exchanges and processing after the first stage of QoS routing.

\section{References}

1. Akyildiz, I.F., et al.: A survey on wireless multimedia sensor networks. Computer Network (2006)

2. Akkaya, K., Younis, M.: A survey of routing protocols in wireless sensor networks, Ad Hoc Network (Elsevier) (2005)

3. Kulkarni, P., Ganesan, D., Shenoy, P.: The Case for Multi-tier Camera Sensor Networks. In: NOSSDAV. Proceedings of the Fifteenth International Workshop on Network and Operating Systems Support for Digital Audio and Video (2005) 